Einführung in das Schwerpunktthema

\title{
Ökonomie der Nachhaltigkeit oder „Nachhaltigkeit" der Ökonomie?
}

A

Von Ulrich Petschow und Jan Nill usgehend vom Brundtland-Bericht 1987 hat das Konzept der Nachhaltigen Entwicklung eine steile Karriere genommen. In Deutschland wurde die konzeptionelle Weiterentwicklung im wesentlichen von verschiedenen Enquete-Kommissionen vorangetrieben. Die Enquete-Kommission des 12. Bundestages entwickelte vier Regeln zur Konkretisierung der ökologischen Dimension der Nachhaltigkeit (1). Zugleich wurde Nachhaltigkeit zunehmend als dreidimensionales Konzept begriffen, umstritten blieb jedoch die Ausgestaltung der und das Verhältnis zwischen den Dimensionen. Hierzu hat die Enquete-Kommission des 13. Bundestages in ihrem Abschlußbericht eine Position formuliert, die die Diskussion zuspitzt (2). Sie begreift Nachhaltigkeitspolitik breit als Gesellschaftspolitik mit eigenständiger ökonomischer und sozialer Dimension, die den gleichen Rang haben wie die ökologische. Ihre ökonomischen Regeln fokussieren auf eine der Eigenverantwortung der Individuen entsprechende Wirtschaftsordnung, die Wirkung des Preismechanismus, einen geeigneten Rahmen für funktionsfähige Märkte und den Erhalt der ökonomischen Leistungsfähigkeit (S. 48, siehe auch die Auszüge aus ihrem Bericht auf S. 12-14.

Sind diese Bedingungen für die „Nachhaltigkeit“ der Ökonomie mit einer Ökonomie der Nachhaltigkeit verträglich, die von den ökologischen Grenzen des Wirtschaftens ausgeht? Ein Kern der Fragestellung nach der ökonomischen Dimension der Nachhaltigkeit ist die Klärung des Verhältnisses zwischen ökologischem und ökonomischem System. Eine historische Einordnung ist hier hilfreich.

\section{Eine historische Perspektive}

Die langfristige wirtschaftliche Entwicklung ist an die jeweils verfügbare Ressourcenausstattung gebunden, wie z.B. Sieferle eindrücklich deutlich gemacht hat (3). In historischer Perspektive hatten gerade die Begrenzungen des Energiesystems Einfluß auf die Entwicklungsmöglichkeiten der Gesellschaften. Innerhalb dieser Grenzen waren jedoch eine Vielzahl von gesellschaftlichen Zuständen möglich, die in der jeweiligen gesellschaftlichen Interpretation als nachhaltig interpretiert werden konnten. Im flächengebundenen solaren Energiesystem der landwirtschaftlichen Produktion waren die Begrenzungen der gesellschaftlichen Handlungsmöglichkeiten durch die Ressourcen weitgehend spïrbar, eine Übernutzung der Ressourcen fuihrte zu direkten Rückkoppelungen an das gesellschaftliche System, wie nicht zuletzt die Herkunft des Begriffes der Nachhaltigkeit aus der Forstwirtschaft andeutet.

Die entscheidende Veränderung fand mit der Industriellen Revolution und der damit verbundenen Entwicklung des fossilen Energiesystems statt. Dies war keineswegs allein eine technische Frage, sondern auch eine Frage gesellschaftlicher Triebkräfte, die auf eine Überwindung der Grenzen zielen. Diese Entwicklung stellte ökonomisch interpretiert eine Veränderung der Knappheitsverhältnisse dar und fïhrte so zu einer fundamentalen Veränderung der relativen Preise. Sie hatte eine massive Expansion der wirtschaftlichen Aktivitäten zur Folge.

Unterstellt man, daß die Prognosen der Klimaforscher der Realität entsprechen, dann sind durch die Nutzung fossiler Energien, inzwischen neue Knappheiten entstanden. Den limitierenden Faktor stellt heute die Aufnahmefähigkeit der Atmosphäre von klimarelevanten (vielfach energiebedingten) Gasen dar. Die Verfiugbarkeit von Energie führt zu einer Übernutzung der Atmosphäre mit kaum absehbaren Folgeschäden. Die Funktionsfähigkeit der Märkte als Vermittler der neuen Knappheiten in diesem Bereich über den Preis ist offenbar nicht (automatisch) gegeben.

\section{Ökonomie und Ökologie}

Vor diesem erweiterten Hintergrund erlangt da s Verhältnis zwischen beiden Systemen vor allem in Fragen nach dem von der Erde tragbaren Ausmaß des ökonomischen Systems und, damit eng verknüpft, Fragen der intergenerativen Gerechtigkeit Bedeutung. Diese wurden insbesondere von der Ökologischen Ökonomie ernst genom- men. Ein überlebensfähiges ökologisch-ökonomisches System ist in Anlehnung an Daly durch drei Bedingungen gekennzeichnet:

1. Ein Ausmaß des Wirtschaftssystems (,,sustainable scale"), das eine Aufrechterhaltung der Life-Support-Function, die die Natur für das System Wirtschaft leistet, garantiert.

2. Eine gerechte Verteilung der Ressourcen und Möglichkeiten, nicht nur zwischen den gegenwärtigen Generationen, sondern auch zwischen heutigen und zukünftigen Menschen.

3. Eine effiziente Allokation der Ressourcen, die das natürliche Kapital angemessen berücksichtigt (4).

Die Interpretation der Enquete-Kommission bleibt vor diesem Hintergrund widersprüchlich. Auf der einen Seite scheint sie dieser Interpretation zuzustimmen: „Die Rahmenbedingungen des Wirtschaftens werden durch die Notwendigkeit des Einfügens in die uns umgebenden Ökosysteme und die Notwendigkeit eines sozialen Ausgleichs gegeben." (S. 38).

Auf der anderen Seite wird dies in Frage gestellt, indem alle Reformbedarfe der Gesellschaft, von der mangelnden Anpassungsfähigkeit des Bildungssystems bis zur Problematik des Generationenvertrages, auf eine Stufe mit der ökologischen Frage gestellt werden. Diese Sichtweise steht in der Tradition der neoklassischen, kapitaltheoretischen Fassung des Nachhaltigkeitsbegriffes, in der die verschiedenen Kapitalarten auch substitutiv genutzt werden können, um das Ziel einer Aufrechterhaltung der gesellschaftlichen Wohlfahrt $\mathrm{zu}$ erreichen; sie wird damit aber nicht unproblematischer (5). Durch die Gleichsetzung unterschiedlicher Veränderungsnotwendigkeiten in der Gesellschaft ist die Enquete-Kommission kaum in der Lage Prioritäten zu setzen - die ökologische Frage muß eben auch gelöst werden. Dementsprechend schwach entwickelt bleiben ihre Vorschläge zur Integration der drei Dimensionen.

Wenn z.B. die mangelnde Anpassungsfähigkeit des Bildungssystems als eine Beeinträchtigung des Nachhaltigkeitsgebotes betrachtet und damit auf eine Stufe mit dem Klimaproblem gestellt wird, dann werden ungleiche Dinge miteinander verglichen, da die zugrundeliegenden Probleme sich im Charakter unterscheiden. Dies kann zu einer falschen Prioritätensetzung führen.

Die Unfähigkeit einer Gesellschaft zum institutionellen Wandel wird spätestens binnen einer Generation spïrbar, sei es aufgrund wachsender Belastungen oder auch abnehmender Wettbe- 
werbsfähigkeit, Sie wird sich entsprechend in gesellschaftlichen Anpassungsprozessen bemerkbar machen, da ein direkter eindeutiger Problemdruck entsteht. Dies ist hingegen z.B. beim Klimaproblem nicht der Fall.

Die Enquete-Kommission identifiziert diese Problematik ebenfalls, kommt aber zu folgendem Schluß: „Selbst wenn man hieraus ein Primat der Umweltpolitik ableiten würde: der Sache wäre damit letztlich doch nicht gedient. Eine ökologisch dominierte Nachhaltigkeitspolitik wird im gesellschaftlichen Abwägungsprozeß immer dann unterliegen, wenn sich andere Problemlagen als unmittelbarer ... erweisen ... Letztlich dürfte nur eine Politik der Integration der drei Dimensionen in der Lage sein, die konzeptionelle Schwäche einer von wirtschaftlichen und sozialen Fragestellungen isolierten Umweltdiskussion zu überwinden“ (S. 31).

\section{- Pfadwechsel entscheidend}

Dieser Einwand ist zwar wichtig, die von der Kommission gezogene Schlußfolgerung ist jedoch problematisch. Am Beispiel der Entwicklung der Energiesysteme wird deutlich, daß es um langfristige Anpassungsprozesse und einen schrittweisen Pfadwechsel geht. Es besteht kaum ein Zweifel, daß eine Reduktion der Treibhausgase in kurzer Zeit zu Verwerfungen im wirtschaftlichen und gesellschaftlichen System führen kann. Dennoch darf eine begrenzte Anpassungsfähigkeit nicht zu einer Politikabstinenz führen. Die wirtschaftlichen und sozialen Systeme haben sich im Zeitablauf als außerordentlich anpassungsfähig erwiesen. Solche Anpassungsleistungen sind allerdings nicht kurzfristig zu erbringen.

Gerade deshalb ist eine Prioritätensetzung erforderlich, die darauf abzielt, in einem definierten Prozeß die neuen Knappheiten zu vermitteln, Ausstiegsfenster zu definieren und die Anpassungsfähigkeit der wirtschaftlichen und sozialen Systeme zu nutzen, um die notwendigen Veränderungen auf den Weg zu bekommen. In diesem Sinne führt die Konzeption der Enquete-Kommission zu einer Fehlorientierung.

\section{Die Beiträge im Überblick}

Die verschiedenen Facetten der skizzierten Kontroverse werden ausgehend vom Konzept der Enquete-Kommission im ersten Teil der folgenden Beiträge kritisch diskutiert. Jürgen Rocblitz setzt sich dabei insbesondere kritisch mit den politischen Implikationen der Konzeption auseinander und präsentiert einen alternativen Regelvorschlag. Paul Klemmer und Frank Beckenbach setzen die Kontroverse auf einer eher theoretischen Ebene fort. Während Klemmer das Konzept der EnqueteKommission verteidigt und als eher noch nicht weitgehend genug einschätzt, nähert sich Beckenbach dem aus einer Perspektive der Ökologischen Ökonomie wesentlich skeptischer.

Der Beitrag von Beckenbach bietet zugleich einen guten Übergang zu der Frage, ob und inwieweit ein derartiger Anpassungsprozeß unter den gegebenen gesellschaftlichen Rahmenbedingungen möglich ist. Inwieweit besitzt das ökonomische System, wie es gegenwärtig existiert und wie es in der Grundvorstellung auch den Regeln zur ökonomischen Nachhaltigkeit der Enquete-Kommission zugrundeliegt, eine inhärente Eigendynamik, die ökologischen Nachhaltigkeitsgrenzen zu überwinden? Impliziert das Ziel der Nachhaltigkeit daher einen Konflikt zwischen ökologischer und ökonomischer Dimension? Was kann dies in der Konsequenz für das Wirtschaftssystem bedeuten? Auch bei diesen Fragestellungen ist eine dynamische Perspektive hilfreich.

\section{- Vermarktlichung der Gesellschaften?}

Gegenwärtig dringen marktorientierte Herangehensweisen immer stärker in Bereiche ein, die bislang anderen Formen des Wirtschaftens vorbehalten waren. Eine umfassende Transformation des Wirtschaftens hin zu marktbasierten Austauschprozessen ist festzustellen. Dies stellt zugleich eine Überwindung der gesellschaftlichen Grenzen dar. In den sog. ,normintegrierten Gesellschaften" (Sieferle) waren gerade auch die wirtschaftlichen Handlungen der Akteure durch ein kulturelles Zentrum eingegrenzt (bzw. eingebettet) und die Reichweite der wirtschaftlichen Handlungen definiert. Ist dies nicht mehr der Fall, so besteht ein wesentliches Problem darin, ,den teils rasanten technischen und ökonomischen Wandel mit den viel trägeren traditionellen sozio-politischen und soziokulturellen Strukturen sowie den ökologisch natïrlichen Prozessen, die wiederum eigene Gesetzmäßigkeiten und Geschwindigkeiten kennen, in Übereinstimmung zu bringen" (S. 29). Leider wird von der Kommission die Frage nach den Triebkräften dieser zunehmenden Beschleunigung und den Konsequenzen hieraus nicht gestellt, obwohl dies im Sinne einer Politik der Nachhaltigkeit ein entscheidender Faktor wäre.
Dies tun dafür die Beiträge im zweiten Teil dieses Schwerpunkts. Fritz Söllner verweist in diesem Zusammenhang vor allem auf den monetären Bereich und schlägt daher vor, zur Gewährleistung der Nachhaltigkeit den Geldschöpfungsmechanismus zu verändern. Für Carola Möller und Ulla Peters verweist die ökonomische Dimension der Nachhaltigkeit hingegen auf den von den derzeitigen ökonomischen Konzepten vernachlässigten Bereich der gemeinwesenorientierten Ökonomie. Abschließend ordnet Hans Diefenbacher diesen Vorschlag historisch ein und versucht, Kriterien für die Sinnhaftigkeit einer stärkeren Orientierung ökonomischer Nachhaltigkeitsregeln auf diese Dimension des Wirtschaftens abzuleiten.

Wir hoffen, mit den Beiträgen dieses Schwerpunkts einen wesentlichen Impuls für die von der Enquete-Kommission selbst eingeforderte Weiterfiihrung der Diskussion (S. 33f) zu geben. Selbst wenn damit noch keine ausgereiften alternativen oder umfassenderen Konzeptionen der ökonomischen Dimension der Nachhaltigkeit präsentiert werden, so wird zumindest gezeigt, daß alternative „Diskussionspfade“ hierfür nötig und möglich sind.

\section{Anmerkungen}

(1)Enquete-Kommission "Schutz des Menschen und der Umwelt" des 12. Deutschen Bundestages: Die Industriegesellschaft gestalten - Perspektiven für einen nachhaltigen Umgang mit Stoff- und Materialströmen. Economica Verlag, Bonn 1994.

(2) Enquete-Kommission "Schutz des Menschen und der Umwelt" des 13. Deutschen Bundestages: Konzept Nachhaltigkeit. Vom Leitbild zur Umsetzung. Deutscher Bundestag, Referat für Öffentlichkeitsarbeit, Zur Sache 4/98.

(3) Sieferle, Rolf Peter: Rückblick auf die Natur. Eine Geschichte des Menschen und seiner Umwelt. Luchterhand, München 1997.

(4) vgl. z.B. Daly, Herman: Allocation, Distribution and Scale. In: Ecological Economics 1992, S. 185 - 193.

(5) Zu einer aktuellen theoretischen Diskussion des Nachhaltigkeitskonzepts aus einer eher kapitaltheoretischen Perspektive vgl. David Pearce/ Giles Atkinson: The Concept of Sustainable Development: An Evaluation of its Usefulness ten Years after Brundtland. CSERGE Working Paper PA 1998-02.

\section{Die Autoren}

Ulrich Pelschow und Jan Nill sind Mitarbeiter im IÖW. Kontakt: IÖW, Giesebrechtstr. 13, 10629 Berlin, Tel. 030/ 8845 94-0, Fax 030/882 54 39, e-mail: Ulrich.Peischow@ioew.b.eunet.de 
(c) 20I0 Authors; licensee IÖW and oekom verlag. This is an article distributed under the terms of the Creative Commons Attribution Non-Commercial No Derivates License (http://creativecommons.org/licenses/by-nc-nd/3.o/), which permits unrestricted use, distribution, and reproduction in any medium, provided the original work is properly cited. 